\title{
MIGRACIÓN DE RETORNO Y EXPERIENCIAS DE REINSERCIÓN EN LA ZONA METROPOLITANA DE LA CIUDAD DE MÉXICO
}

\author{
Liliana Rivera Sánchez*
}

\begin{abstract}
Este artículo tiene como objetivo reflexionar sobre las condiciones contemporáneas del retorno de migrantes mexicanos de Estados Unidos a México, particularmente sobre el retorno y la inserción en contextos urbanos. Por un lado, se analizan algunos datos sociodemográficos de los retornados provenientes de los censos de población de los años 2000 y 2010. Por otro lado, se presentan fragmentos de relatos biográficos acerca de la experiencia de retorno durante la última década, para mostrar cuáles son las condiciones en las que se reinsertan tanto social como laboralmente, cómo se construyen las expectativas de re-emigración (internacional/interna) y cómo los sujetos diseñan ciertas estrategias de relocalización tanto social como laboral en los contextos urbanos de retorno.
\end{abstract}

Palabras clave: migración de retorno, reinserción social, reinserción laboral, Estados Unidos, México.

\section{Las premisas básicas: una introducción al estudio del retorno ${ }^{1}$}

A lo largo de varias décadas, los estudios sobre los migrantes retornados de Estados Unidos a México se han concentrado fundamentalmente en las áreas rurales, sin duda porque hasta mediados de la década del ochenta la mayoría de los migrantes mexicanos con destino a Estados Unidos, procedían de las localidades rurales del país. No obstante, la migración internacional ha generado otros patrones de migración y constituido nuevos perfiles de

\footnotetext{
* Investigadora del Centro Regional de Investigaciones Multidisciplinarias de la Universidad Nacional Autónoma de México. Cuernavaca, Morelos/México.

1 Los hallazgos de investigación que se presentan en este artículo son resultado del proyecto CONACYT No. 105357 “Movilidades y establecimiento. Migrantes retornados en Nezahualcóyotl, ¿Quemar las naves o re-emigrar?" (2010-2013).
} 
migrantes, los cuales incluyen tanto a mujeres que emigran sin necesariamente ir como acompañantes de varones, así como a inmigrantes procedentes de ciudades; entre otros cambios, se puede mencionar también nuevas regiones de emigración en México y nuevos destinos en Estados Unidos, así como variaciones relacionadas con el perfil demográfico de los inmigrantes contemporáneos ${ }^{2}$. Los migrantes que salen de las ciudades mexicanas hacia Estados Unidos no necesariamente son de origen urbano, algunos tienen como lugar de origen una localidad rural, es decir han experimentado previamente migración interna y desde allí han emprendido su viaje al norte. Hoy en día, las ciudades mexicanas pueden ser lugares de origen y/o lugares de salida de la migración internacional y entonces, tales interconexiones de trayectos migratorios generan también un panorama más complejo para analizar tanto la salida de migrantes, como el retorno ${ }^{3}$. Las reflexiones conceptuales sobre el retorno migratorio y los hallazgos de investigación que se presentan en este artículo tienen como objetivo mover el lente de los estudios realizados en la región tradicional de la migración México-Estados Unidos, y abordar algunos aspectos de las experiencias contemporáneas del retorno migratorio en contextos urbano-metropolitanos, cuyo marco de referencia es la crisis de los mercados de trabajo global. Adicionalmente, en términos metodológicos, los resultados de investigación presentados pretenden tomar distancia de las investigaciones que consideran como unidades de análisis y de referencia sólo un lugar de origen y un lugar de destino para estudiar el proceso migratorio, y en consecuencia asumen que el retorno ocurre necesariamente al lugar de origen. Esa estrategia ha conducido a observar exclusivamente a las personas procedentes del mismo lugar, estudiarlas como "un grupo étnico", un grupo local, o una comunidad, esencializando el lugar de procedencia como un marcador de homogenización social y cultural, que si bien es relevante considerarlo por el efecto que tiene en la constitución de redes sociales y vínculos, también es crucial no esencializarlo. Esto hace suponer a priori que los migrantes -en este caso los retornados-, comparten necesariamente trayectorias y prácticas culturales asociadas a la localidad desde la cual se desplazaron.

En consecuencia, se plantea estudiar a los retornados de Estados Unidos a Nezahualcóyotl, a partir de analizar las motivaciones para regresar,

\footnotetext{
2 DURAND, Jorge; MASSEY, Douglas. Clandestinos. Migración México-Estados Unidos en los albores del siglo XXI.

${ }^{3}$ Cf. RIVERA-SÁNCHEZ, Liliana; LOZANO, Fernando. Los contextos de salida urbanos y rurales y la organización social de la migración. Además, cf. RIVERA-SÁNCHEZ, Liliana. El eslabón urbano en el trayecto interno del circuito migratorio Mixteca-Nueva York-Mixteca: Los migrantes de Nezahualcóyotl, Estado de México.
} 
su trayectoria migratoria y experiencia de retorno, así como los procesos de reinserción laboral y social. Se reconoce que, aun en un mismo contexto, los efectos del retorno son diferenciados, tomando en cuenta el capital social, la posición en los campos sociales, y la relación con los contextos y las condiciones que median su retorno (devueltos, repatriados, deportados, o "voluntarios") entre otros factores de diferenciación social. No se pretende clasificar a los retornados en alguna de las dos categorías sociales con las que comúnmente se han etiquetado: "exitosos" y "fracasados"4, sino que la misma escala de la ciudad -como sitio de prueba para la investigación- inserta en la zona metropolitana ${ }^{5}$ y atravesada por movilidades diversas, ofrecerá elementos para complejizar los resultados y la misma experiencia de retorno internacional.

En síntesis, se propone entender el contexto de retorno y en éste el papel de los retornados, como agentes sociales que median entre las condiciones estructurales y las condiciones subjetivas/objetivas que se entretejen en la experiencia, para diseñar estrategias de reinserción. Por lo tanto, la experiencia de retorno se analiza a lo largo del artículo como parte del proceso migratorio y no como una conclusión definitiva del proyecto como migrante; esto es, el retorno no necesariamente es definitivo y permanente, sino una fase del proceso migratorio, que tiene efectos (al igual que la emigración) sobre las personas y los lugares ${ }^{6}$.

Así, el estudio que sustenta el artículo se desarrolló en el municipio de Nezahualcóyotl como el lugar de retorno, el cual puede ser también, en el itinerario de los migrantes, el lugar de origen, el lugar de salida (para inmigrantes que experimentaron previamente migración interna además de la internacional), un lugar de paso o uno para realizar una escala temporal antes de regresar a la localidad de origen, re-emigrar a un destino interno, o bien hacerlo nuevamente a uno internacional? .

Finalmente, en este documento subyace el presupuesto de que un estudio sobre la reinserción social y laboral de los migrantes retornados a un espacio urbano podría contribuir a entender los efectos sobre:

\footnotetext{
${ }^{4}$ Cf. CASSARINO, Jean-Pierre. Theorising Return Migration: The Conceptual Approach to Return Migrants Revisited; DURAND, Jorge. Ensayo teórico sobre la migración de retorno. El principio del rendimiento decreciente.

${ }^{5}$ Para una explicación sobre las escalas y el papel de las ciudades (con presencia de población inmigrante) en los procesos de incorporación o inserción laboral y social, cf. GLICK-SCHILLER, Nina; CAGLAR, Ayse (eds.). Locating Migration. Rescaling Cities and Migrants.

${ }^{6}$ Cf. RIVERA-SÁNCHEZ, Liliana. ¿Quiénes son los retornados? Apuntes sobre el migrante retornado en el México contemporáneo.

${ }^{7}$ Cf. RIVERA-SÁNCHEZ, Liliana. El eslabón..., op. cit.
} 
- La probabilidad de conseguir un empleo en el mercado de trabajo local, las formas como opera el capital social, la escolaridad y, en general las habilidades y destrezas adquiridas en la estancia como inmigrantes internacionales, al regresar a México. Es decir, poner a prueba la tesis sobre el capital humano acumulado para observar el papel que juega en el proceso de reinserción laboral.

- Las relaciones familiares y personales, el proceso de reajuste a otra dinámica social en un contexto (ahora contexto de retorno) que sin duda ha cambiado, no obstante que el migrante haya salido de ese mismo sitio. Es decir, el efecto de readaptación o reincorporación, de ajuste de expectativas o de conflicto, que generan las movilidades humanas.

- La relación movilidad-establecimiento, particularmente el efecto que el retorno de los migrantes internacionales podría tener en un contexto urbano, localizado en la zona metropolitana de la Ciudad de México. Esto es, poner a prueba la hipótesis de que en México, el efecto de la migración internacional (y particularmente de los retornados) "se diluye" en el contexto urbano, y sólo se refleja de manera nítida en las localidades rurales, a través de cambios evidentes en los paisajes locales y la dinámica comunitaria.

A continuación se presenta una breve revisión acerca de cómo se ha explicado el retorno migratorio internacional en la literatura del campo de estudio de las migraciones, con el objetivo de enmarcar teóricamente las reflexiones ulteriores.

\section{El retorno en la literatura del campo de estudio de las migraciones}

Grosso modo, el retorno de los migrantes laborales ha sido explicado desde cinco perspectivas teóricas, las que también explican el inicio del proceso migratorio. La perspectiva neoclásica y la así llamada New Economics of Labour Migration han explicado el fenómeno como parte de las decisiones racionales, económicas, que toman los individuos para maximizar su beneficio. Basada particularmente en el presupuesto de la disparidad salarial como el motor que conduce a tomar la decisión de emigrar ${ }^{8}$, la perspectiva neoclásica explica el retorno entonces como una consecuencia de la experiencia de fracaso del migrante, o bien porque las expectativas sobre su capital humano no fueron

\footnotetext{
${ }^{8}$ Cf. TODARO, Michael. A model of Labour Migration and urban employment in less development countries.
} 
valoradas como se esperaba ${ }^{9}$. Mientras, la Nueva Economía de la migración laboral supone que, si bien no se trata de un fracaso de los individuos que tomaron la decisión de migrar, ni de una falla en el cálculo realizado sobre su capital humano, el retorno se explica, por el contrario, como la conclusión de las metas económicas que el migrante - conjuntamente con los demás miembros de su familia - había construido y valorado en el momento en el que se tomó la decisión de la emigración de uno los miembros de la familia. Stark ${ }^{10}$ considera que, el retorno es justamente un resultado natural del cálculo realizado al emprender el desplazamiento, lo que implica un resultado exitoso de su proyecto; es decir, supone que la conclusión del proceso indica que el migrante por un lado, ha realizado ahorros, y por el otro, que ha enviado recursos a su familia en el transcurso de su estancia migratoria. Los dos aspectos anteriores contribuyen tanto a diversificar las fuentes de ingreso del hogar, como a inyectar dinamismo en la economía local del país de origen y de destino. En esa línea de reflexión, Taylor ${ }^{11}$ sugiere que, al retorno no sólo se han consumado las metas del migrante y su familia, sino que este individuo que toma la decisión de regresar ha adquirido habilidades y conocimiento que puede potenciar en su lugar de nueva inserción y tal consideración podría aumentar las probabilidades del retorno mismo.

No obstante que ambas perspectivas observan motivaciones económicas tanto para salir como para retornar, no refieren en sus consideraciones sobre "fracaso" o "éxito" - como señala Cassarino ${ }^{12}$ - a dónde regresan los migrantes, es decir no ven más allá de las consideraciones sobre el individuo y el hogar y, eventualmente consideran las posibilidades reales de una mejor reinserción laboral debido a las habilidades y destrezas adquiridas en el lugar de destino. En este sentido, el enfoque estructural sobre la migración de retorno introduce el contexto como una variable relevante a considerar, fundamentalmente el contexto institucional y social del país de origen, los llamados factores situacionales y estructurales que condicionan el resultado del retorno y las motivaciones para tomar la decisión de regresar. Así, se sugiere que en la medida en que tal acto de volver implica una readaptación a otro ambiente, el mismo podría conducir también a una re-emigración, en el caso de que tal ajuste a ese contexto no se consiguiera. De tal forma que, los factores contextuales juegan un papel central, no sólo las habilidades adquiridas y el dinero ahorrado por el migrante para retornar. Es decir, el impacto de los retornados no necesariamente es visto

\footnotetext{
${ }_{9}^{9}$ Cf. CASSARINO, op. cit.; DURAND, op. cit.

${ }^{10}$ STARK, Olson. The Migration of Labour.

${ }^{11}$ TAYLOR, J. Edward. Development strategy, Employment and Migration: Insight from Models.

${ }^{12}$ CASSARINO, op. cit., p. 257.
} 
como uno con efectos multiplicadores de desarrollo, en la medida en que está mediado justamente por las condiciones diferenciales de los contextos. Esto es, que la relación asimétrica entre los países de origen y destino podría imponerse también al retorno y no ofrecer las condiciones suficientes para una movilidad social ascendente y quizás ni siquiera para una readaptación o reajuste de expectativas sociales, porque suponen que no existe un proceso de intercambio de recursos permanente entre ambos países ${ }^{13}$. Pareciera que el inmigrante, durante el tiempo de estancia en un destino internacional, se mantuviera desconectado del ambiente social y de la información relativa al lugar de origen, lo cual implica que no se reconoce la circulación y la movilización de recursos entre estos dos contextos (de origen y de recepción).

En suma, desde el enfoque estructural, y desde sus diversas vertientes teóricas, se propone, en términos generales, las siguientes variables básicas para estudiar el retorno: el tiempo de estancia como migrante, la escolaridad, la trayectoria laboral, las habilidades adquiridas y las características del contexto institucional y social del país de origen.

Desde la perspectiva de redes sociales se parte del supuesto que existe tal movilización de recursos, la cual no aparece claramente como una posibilidad desde el enfoque estructural, y que justamente a través de las redes sociales y de los vínculos que se mantienen entre las sociedades de origen y destino es que se hace posible el retorno. Las estructuras sociales sobre las que descansan las redes y su constante intercambio conforman ciertos capitales que son útiles para emigrar y también para retornar. No obstante, no todos los migrantes tienen acceso homogéneo a tal capital, ni siquiera a insertarse por igual en las redes, y luego, las posibilidades y condiciones del retorno también son heterogéneas y dependerán del acceso al capital y, por supuesto, de las posiciones de los actores en los campos sociales. Dos consideraciones analíticas son relevantes en la perspectiva de redes para identificar las unidades de análisis; una es que los individuos son actores sociales involucrados en redes de relaciones; y otra, es que es necesario tomar en cuenta que tales redes conforman diversos tipos de agregados, y/o organizaciones, y luego, que tales plantean a su vez diferentes niveles de involucramiento, orientación y estrategias de relación ${ }^{14}$. Finalmente, la perspectiva de redes plantea no estudiar a los retornados sólo como un agregado o un grupo, sino como individuos insertos en redes de relaciones que influyen también sus acciones y decisiones. Así, la movilización de recursos a través de las redes sociales es no sólo de recursos tangibles, sino

\footnotetext{
${ }^{13}$ Cf. SINGER, Paul. Economía Política de la Urbanización.

${ }^{14}$ Cf. CASSARINO, op. cit.
} 
de recursos intangibles, como puede ser el capital social ${ }^{15}$. Es decir, desde la perspectiva de redes resulta relevante estudiar las relaciones entre los retornados y los inmigrantes que no retornan, pero también de manera paralela dar cuenta de la movilización de recursos que ocurre a través de las redes que atraviesan las fronteras ${ }^{16}$.

Durand ${ }^{17}$ ha planteado la posibilidad de que la teoría del capital social pueda explicar también las probabilidades del retorno, así como ha explicado la salida acumulada de migrantes de un lugar de origen hacia un mismo lugar de destino. Es decir, la teoría de la causalidad acumulativa, según Massey, Goldring y Durand ${ }^{18}$, llevada al contexto de retorno, podría decir que "a mayor experiencia acumulada de retorno en la familia, la comunidad y el país de origen, mayores posibilidades de que se dé el retorno a nivel personal"19.

Por su parte, la perspectiva transnacional considera que la migración de retorno es parte del proceso migratorio y se inscribe en la mirada sistémica y compleja del mismo proceso, el cual incluye relaciones económicas, sociales y culturales entre las sociedades de origen y las de destino, mediadas por un constante intercambio de recursos, dinero, bienes, ideas, información y valores ${ }^{20}$. Desde esta perspectiva, el retorno debe ser estudiado considerando las características y modalidades de involucramiento tanto en los lugares de origen como en los de destino, pero además tomando en cuenta que el ambiente social y las estructuras institucionales de ambas sociedades delinean el retorno, el cual puede ocurrir a un tercer lugar que no sea necesariamente el lugar de origen y puede tratarse incluso de una escala en el trayecto de la vida como inmigrante ${ }^{21}$.

Así, el retorno aparece, desde las perspectivas transnacional y global de las migraciones, como la contraparte de los estudios sobre los procesos de integración/incorporación en las sociedades de destino, pero analizado como una pieza de la experiencia de atravesar diversas fronteras y circular constantemente por diferentes lugares, finalmente como parte de la experiencia de las movilidades entre sociedades desiguales ${ }^{22}$.

\footnotetext{
${ }^{15}$ Cf. DURAND, op. cit.; CASSARINO, op. cit.

${ }^{16}$ CASSARINO, op. cit.; DURAND, MASSEY, op. cit.

${ }^{17}$ DURAND, op. cit.

${ }^{18}$ MASSEY, Douglas; GOLDRING, Luin; DURAND, Jorge. Continuities in Transnational Migration: An Analysis of Nineteen Mexican Communities.

${ }^{19}$ DURAND, op. cit., p. 110.

${ }^{20}$ LEVITT, Peggy; SORENSEN, Nyberg. The Transnational Turn in Migration Studies.

21 RIVERA-SÁNCHEZ, Liliana. El eslabón urbano en el trayecto interno del circuito migratorio Mixteca-Nueva York-Mixteca: Los migrantes de Nezahualcóyotl, Estado de México.

22 GLICK-SCHILLER, Nina. Transnational Social Fields and Imperialism: Bringing a theory of power to the transnational studies.
} 
En el siguiente apartado delinearemos el sitio de prueba de nuestra investigación para entender las modalidades de la reinserción social y laboral de los migrantes de retorno.

\section{El municipio de Nezahualcóyotl, Estado de México, el sitio de prueba para la investigación}

Nezahualcóyotl fue fundado el 3 de abril de 1963. Los terrenos que hoy conforman el municipio de Nezahualcóyotl pertenecen históricamente al Lago de Texсосо en la zona metropolitana de la ciudad de México, el cual fue desecado entre 1912 y 1932. Una vez que concluyó el proceso de desecación inició la historia de los asentamientos en la región de Texcoco. Los terrenos de propiedad federal empezaron a ser vendidos a bajo costo, pues carecían de servicios públicos básicos.

En la década de los cuarenta con la construcción del bordo de Xochiaca y el túnel de Tequixquiac, se generó la afluencia masiva de habitantes de la ciudad de México, en general de otros municipios metropolitanos y de otras entidades del país, que buscaban asentarse en la zona metropolitana y que contaban con pocos recursos para hacerlo. Este proceso dio inicio a la formación de las primeras colonias, entre las que se encuentran Juárez Pantitlán, México, El Sol, El Barrio de Juárez o San Juan, por mencionar algunas de las tradicionales. En 1953 ya existían 13 colonias proletarias sumamente pobladas. Seis años más tarde, en 1959 había 33 colonias. La explosión demográfica y la falta de servicios e infraestructura urbana generaron una gran organización de los pobladores, quienes empezaron a demandar que las colonias se organizaran en fraccionamientos habitacionales con el objetivo de generar una estructura de representación en la gestión urbana del territorio, y exigir a través de esta estructura la atención gubernamental, no solo a nivel federal, sino del Estado de México.

La reorganización de las colonias en el ex-vaso de Texcoco provocó cierto proceso de reordenamiento territorial y, en 1963 uno de los cuatro principales fraccionamientos de colonias de la región Texcoco fue separado administrativamente del municipio de Chimalhuacán, constituyéndose entonces como un municipio independiente, bajo el nombre de Nezahualcóyotl. No obstante, el proceso de lotificación y regularización de los terrenos ha sido largo, y hasta la década de los noventa la regularización de la tenencia de la tierra, el uso del suelo y la dotación de servicios públicos ha sido una demanda constante de la población del municipio.

Actualmente, el municipio se encuentra inserto en la zona conurbada de la Ciudad de México, en la porción oriental del Valle de México. Está 
compuesto por dos localidades, a lo largo de 86 colonias urbanas. El total de la población en 2010 fue de 1,107,931 habitantes, según el Censo de Población y Vivienda del año 2010. Por su ubicación en la zona metropolitana y el hecho de ser un municipio de relativa reciente creación, los habitantes provienen de casi todos los estados del país; es decir, es producto por un lado, de la migración interna (del campo a la ciudad) hacia la zona metropolitana del Valle de México y, por el otro, también de cierto proceso de precarización de los habitantes de la Ciudad de México y otros municipios metropolitanos del Estado de México que, entre 1970 y 1980 vieron mermadas sus condiciones de vida, desplazándose hacia la periferia de la zona metropolitana. Entre 1970 y 1980 la tasa de crecimiento poblacional fue de 8.7\%, doblando prácticamente el promedio de crecimiento estatal. Pero a partir de la década de los ochenta el decrecimiento ha sido constante ${ }^{23}$.

\section{Apuntes demográficos para delinear un contexto de retorno}

El municipio de Nezahualcóyotl, al igual que otros municipios metropolitanos del Estado de México, ha sido lugar de destino recurrente para migrantes procedentes de distintos estados y municipios del país, así como del Distrito Federal. Todos los estados del país cuentan con inmigrantes radicados en Nezahualcóyotl, como se ha señalado previamente. Al mismo tiempo, el número de emigrantes a Estados Unidos que salen de Nezahualcóyotl había tenido un crecimiento ascendente sostenido hasta el año 2010. Lo mismo había ocurrido en el caso de la población que retorna de Estados Unidos a este municipio. No obstante, la población total que reside en el municipio de Nezahualcóyotl ha descendido en las últimas tres décadas ${ }^{24}$, como consecuencia de un ensanchamiento del área metropolitana y luego un proceso de relocalización de la población en la zona conurbada con el Distrito Federal, lo que ha implicado ciertos desplazamientos poblacionales hacia municipios aledaños, tales como Chimalhuacán, Ixtapaluca, Los Reyes, y Chalco Solidaridad, en el Estado de México. Un dato relevante de la población que residía en el año 2010 en Nezahualcóyotl es que 60\% del total no era originaria del Estado de México, estado donde se localiza este municipio. El primer grupo de personas no nacidas en el Estado de México estaba integrado por quienes son originarios del Distrito Federal, los que en 2010 representaron 40\% del total de la población, un porcentaje

\footnotetext{
${ }^{23}$ Cf. INEGI. Censo de Población y Vivienda 2010.

${ }^{24}$ En el año 2010, el municipio de Nezahualcóyotl registró una población total de 1,107,931 habitantes, y en el año 2000 había registrado 1,225,972 habitantes, es decir tuvo un decremento de nueve puntos porcentuales en una década. Cf. INEGI, Censo de Población y Vivienda, 2000 y 2010.
} 
similar al de los nacidos en el Estado de México. El 20\% restante se distribuyó entre quienes nacieron en otras entidades del país. En orden de relevancia se encuentran quienes nacieron en el estado de Puebla, luego le siguen los nacidos en Veracruz, en Oaxaca y Michoacán (por mencionar los principales), todos ellos con una representación porcentual menor al 5\% respecto del total de la población ${ }^{25}$.

Los datos anteriormente presentados muestran que el municipio de Nezahualcóyotl experimenta una intensa movilidad poblacional, en diversas modalidades y de manera simultánea: emigración hacia otros municipios de la zona metropolitana y hacia el Distrito Federal, principalmente, además de hacia destinos internacionales (a Estados Unidos fundamentalmente) y sigue manteniendo una dinámica de inmigración constante de nuevos pobladores procedentes del interior del país ${ }^{26}$, además de ser receptor de migrantes de retorno internacional.

En el caso de los emigrantes a Estados Unidos que salieron de Nezahualcóyotl en la última década es notable el descenso que registra el Censo de Población entre el año 2000 y el 2010. Entre 2005 y 2010 salieron 63\% menos personas con destino a Estados Unidos que quienes salieron entre 1995 y el año 2000. Por el contrario, entre los migrantes de retorno del quinquenio 1995-2000 y el registrado en el periodo 2005-2010 se observa un importante incremento, los retornados en este último quinquenio representan 40\% más. Lo anterior significa que regresaron $40 \%$ más migrantes de Estados Unidos a Nezahualcóyotl entre 2005-2010 que los que regresaron en el quinquenio 1995-2000. Pero sin duda el incremento más significativo es el que se registra comparando el número de personas retornadas en el quinquenio entre 2000 y 2005, respecto de quienes volvieron en el quinquenio inmediato siguiente (2005-2010), la diferencia es de 66\% más en éste último, lo cual muestra sin duda que, el retorno migratorio a Nezahualcóyotl se ha incrementado durante la última década y con mayor énfasis entre 2005-2010, periodo que coincide con la agudización de la crisis económica en Estados Unidos y luego la contracción de los mercados de trabajo ${ }^{27}$.

\footnotetext{
${ }^{25}$ Cf. INEGI. Censo de Población y Vivienda 2010.

${ }^{26}$ Destacan los inmigrantes en Nezahualcóyotl llegados en los últimos cinco años procedentes de los municipios metropolitanos, tales como Ecatepec de Morelos, Chimalhuacán, Ixtapaluca, y Chalco del Estado de México, así como de las delegaciones Iztapalapa, Gustavo A. Madero y Venustiano Carranza, del Distrito Federal (ibidem).

${ }^{27}$ Según el Instituto Nacional de Estadística y Geografía (2012), el saldo neto migratorio internacional en México, al primer trimestre del año 2012 es prácticamente nulo. De acuerdo con el análisis de la serie histórica, durante el año 2010, en México se perdió menos población por efecto de la migración internacional (8.5 por cada 10 mil) y aunque se recuperó para 2011 (11.6), la tasa de emigración a Estados Unidos todavía se encuentra por debajo de la tasa promedio registrada para
} 
Asimismo, este incremento en el volumen de retornados en el último quinquenio respecto de los dos previos, podría incluso estar reflejando el efecto que ha tenido la implementación de ciertos dispositivos de una política migratoria restrictiva en algunos estados fronterizos de Estados Unidos (como parte del programa denominado Comunidades Seguras), políticas que han generado condiciones adversas para la población inmigrante indocumentada en aquél país, independientemente de los años de estadía y la experiencia como inmigrante (redadas en espacios laborales, deportaciones y repatriaciones, disposiciones legales que restringen algunos derechos sociales, entre otros), el reforzamiento de la frontera entre México y Estados Unidos, así como el efecto de la saturación de los mercados de trabajo de mano de obra no calificada en los países centrales (sobre todo en los sectores de los servicios y la manufactura), como lo han señalado algunos especialistas a propósito de la dinámica de los mercados de trabajo estadounidenses desde $2008^{28}$.

Por lo anterior, se vuelve aún más relevante hurgar en las modalidades que adquiere la reinserción laboral y social de los migrantes retornados contemporáneos en la zona metropolitana de la ciudad de México, donde el mercado de trabajo informal ha ido adquiriendo mayor peso y ensanchando su tamaño en los últimos años ${ }^{29}$.

Por otro lado, analizando de manera comparativa el perfil de los retornados del quinquenio 1995-2000 y del periodo 2005-2010 destacan las siguientes características: las personas retornadas de Estados Unidos a Nezahualcóyotl, según datos del año 2000, se concentraban en los segmentos poblacionales que incluyen, además de los niños entre los 5 y 9 años, a los adultos entre los 20 y 39 años de edad, y particularmente en el segmento entre los 25 y 29 años de edad de manera significativa; es decir, los retornados se encuentran en plena edad productiva y aun en el rango de edad considerado como joven (menores de 30 años). Se trataba mayoritariamente de hombres, aunque también se detectó un número relevante de mujeres entre las edades de 20 y 34 años. En suma, los migrantes retornados en Nezahualcóyotl registrados en el año 2000 se distribuyeron por sexo: $62 \%$ hombres y $38 \%$ mujeres, y tenían en promedio 30 años de edad. En el año 2010, la estructura por sexo y edad de la población retornada a Nezahualcóyotl se distribuyó 65 $\%$ hombres y $35 \%$ mujeres, disminuyendo ligeramente la participación de

el año 2009, que fue de 13.9. Cf. INEGI. Boletín de Prensa 204/12, México, 5 de junio de 2012.

28 DURAND, Jorge. Conferencia pública. Seminario Permanente sobre Migración Internacional. El Colegio de la Frontera Norte, Tijuana, octubre de 2009.

${ }^{29}$ Por razones de espacio, en este artículo no analizaremos las condiciones de empleo y escolaridad de la población retornada, no obstante son variables centrales que permiten explicar sus modalidades de reinserción laboral. 
las mujeres (tres puntos porcentuales) respecto del registro en el año $2000^{30}$. El promedio de edad de los retornados se mantiene en 30 años (mediana de edad) siendo las mujeres más jóvenes que los hombres retornados (una diferencia en promedio de 4 años de edad).

Un cambio en la estructura por edad de la población retornada en Nezahualcóyotl según el registro de 2010 respecto del año 2000, se observa en los rangos de edad en los cuales se concentra: entre los 25 y 39 años de edad en 2010; mientras en el año 2000 se mantenía una fuerte participación entre los 20 y 25 años. Comparativamente, en 2010 se concentran en el rango inmediato superior, pero sobre todo se distribuyen en dos segmentos de edad, tanto en el de 25 a 29 años, como en el de 35 a 39 años, para hombres como mujeres. En síntesis, se puede afirmar que no ha variado significativamente el perfil de edad y sexo de la población retornada en Nezahualcóyotl en los últimos quince años, continúan concentrándose en los rangos de edad productiva, incluyendo uno de los segmentos que caracteriza a la población considerada como joven (25-29 años), no obstante que se observa una tendencia a concentrarse en los rangos de edad subsecuentes (mayores) en 2010.

Adicionalmente, cabe señalar que 39\% del total de las personas que regresaron de Estados Unidos a Nezahualcóyotl durante el periodo 2005-2010 son originarios del Estado de México y 38\% originarios del Distrito Federal; es decir, $77 \%$ de los retornados son originarios de municipios metropolitanos fundamentalmente, y $23 \%$ provienen de otras entidades, destacando en orden descendente los originarios de los estados de Michoacán, Guerrero, Oaxaca, Puebla y Yucatán, como los principales. Adicionalmente, estas proporciones muestran que al menos $61 \%$ de la población retornada a Nezahualcóyotl tiene también experiencia migratoria interna, además de la internacional. Lo anterior significa que, seis de cada 10 retornados han experimentado al menos dos viajes, uno nacional al mudarse de otra entidad a Nezahualcóyotl, y por lo menos un viaje internacional cuando emigraron a Estados Unidos. Esta experiencia migratoria podría ser también un factor relevante en la constitución de cierto capital para la consecución de un empleo.

\section{Casos prototípicos de inserción laboral y social entre los migrantes de retorno reciente en Nezahualcóyotl}

Para los fines de este artículo, se ha optado metodológicamente por presentar brevemente la narrativa de la trayectoria de los retornados, cuyos casos fueron considerados prototípicos de ciertas modalidades de inserción

${ }^{30}$ INEGI, op. cit. 
laboral y social en Nezahualcóyotl. Estos casos fueron identificados/ construidos al contrastar el conjunto de narrativas vitales recabadas a partir de un diseño de entrevista semi-estructurada, de corte biográfico, que permitió luego diseñar la narrativa de los llamados prototipos ${ }^{31}$. Se construyeron así dos casos prototípicos: el de "reinserción con reemigración" y el de "reinserción con establecimiento" de retornados a Nezahualcóyotl, los cuales se acoplan en términos generales a los perfiles demográficos promedio de los inmigrantes internacionales en Nezahualcóyot ${ }^{32}$. Esta modalidad de investigación longitudinal cualitativa (de corte retrospectivo) utiliza el análisis de las trayectorias para identificar patrones y luego construir/elegir los casos prototípicos, que representan una tendencia o patrón de trayectorias ${ }^{33}$.

En suma se pretende observar a partir de tales casos prototípicos cuáles son las condiciones que hacen posible la reinserción tanto laboral como social en el contexto de retorno y cómo los actores generan estrategias de acomodamiento en esos contextos, y luego cuál es el rol que juega la experiencia como inmigrante en Estados Unidos en este proceso de retorno; finalmente, cuál es el resultado del retorno en estos casos seleccionados en los cuales se observa una intersección entre las condiciones locales y las globales de los mercados de trabajo, y de sociedades que funcionan de manera interconectada. A continuación se presentan los casos prototípicos de retornados en Nezahualcóyotl, cuyos nombres son Luis y Roberto ${ }^{34}$.

Caso 1: LUIS (entrevistado en febrero de 2011 en Nezahualcóyotl) 35 años, soltero, retornado de Estados Unidos a finales de 2008. Originario de Michoacán, inmigrante en Nezahualcóyotl, arribó a la edad de 4 años. Salió de Nezahualcóyotl, Estado de México, en enero de 2002 con destino a Nueva York, a la edad de 27 años. Era soltero, vivía en el mismo hogar que sus padres y una hermana en la Colonia San Agustín, en Nezahualcóyotl; era el mayor de

31 Para una revisión amplia sobre los fundamentos y el uso de esta metodología para el análisis de trayectorias y la construcción de casos prototípicos, cf. PEDREÑO CÁNOVAS, Andrés. Familias inmigrantes: El trabajo de los padres y las estrategias de trabajo de los hijos en las áreas mediterráneas de agricultura intensiva.

32 A lo largo de este artículo se ha usado de manera indistinta las siguientes categorías, para referirse a la misma población: "Inmigrante internacional en Nezahualcóyotl" (considerando solo nacidos en México), "migrante de retorno a Nezahualcóyotl" y simplemente "retornado".

${ }^{3}$ Para mayor referencia acerca del uso e implementación de la trayectoria como una herramienta analítica en los estudios de migración, cf. RIVERA-SÁNCHEZ, Liliana. Las trayectorias en los estudios de migración: Una herramienta para el análisis longitudinal cualitativo.

${ }^{34}$ Los nombres asignados a los actores que dieron pauta para la construcción de los casos prototípicos no son los nombres reales de los retornados entrevistados. Se tomó la decisión de cambiar el nombre para respetar la identidad de los informantes, quienes expresamente otorgaron el permiso para hablar de su experiencia y su vida como inmigrantes en Estados Unidos, y hoy retornados en Nezahualcóyotl, Estado de México. 
tres hermanos, tenía un hermano que radicaba en la ciudad de Nueva York y que llevaba 4 años como inmigrante en aquélla ciudad, cuando Luis decidió emigrar. Es hijo de inmigrantes internos, procedentes del estado de Michoacán, sin experiencia migratoria internacional. Tiene como nivel máximo de estudios el segundo año de secundaria, es decir cursó y aprobó 8 años escolares.

Luis cruzó la frontera en Nogales, Sonora, y desde Arizona viajó en avión directamente a la ciudad de Nueva York, con el plan original de permanecer por dos años para contribuir en la construcción de la casa paterna y solventar los gastos de la familia, no obstante tuvo una estancia de seis años continuos en Estados Unidos, es decir realizó un solo viaje internacional y había realizado previamente uno de migración interna cuando era un niño. Durante los seis años en Estados Unidos vivió 5 años y 9 meses en Nueva York, dos meses en Atlantic City y uno más en Pennsylvania.

Cuando Luis decidió emigrar a Nueva York trabajaba como empleado en una fábrica de embutidos en Nezahualcóyotl, Estado de México, donde se desempeñaba como un obrero general y contaba con seguridad social (aguinaldo, seguro médico, vacaciones), ganaba un poco más del salario mínimo establecido en la zona metropolitana. Emigró solo, orientado por su hermano que estaba en NY, quien le prestó dinero para hacer el viaje, préstamo que rembolsó en un periodo de un año y medio. Los primeros 18 meses de estancia en Estados Unidos, Luis no envió remesas a su familia en Nezahualcóyotl, solo consiguió financiar su estancia y cubrir el préstamo recibido para su viaje (ascendió a 28 mil pesos mexicanos el cruce y viaje por avión hasta NYC). Los siguientes cuatro años y medio, Luis envió remesas mensuales a su familia establecida en Nezahualcóyotl, quienes contaban con un terreno que adquirieron previamente. Las remesas permitieron construir la casa y avanzar en la construcción de un cuarto anexo donde él planea vivir, una vez que lo concluya.

La historia laboral de Luis inicia a la edad de 14 años, como obrero eventual en fábricas en la zona metropolitana, y trabajando como peón albañil conjuntamente con su padre, quien era un maestro albañil. A la edad de 27 años llega a Nueva York y se emplea de inmediato en la misma compañía en la que trabajaba su hermano, como ayudante en el manejo de materiales para la construcción de casas por un periodo de tres años. Su segundo empleo fue en un restaurant, el cual consiguió sin el contacto de su hermano, como ayudante de cocina, pero conservó adicionalmente el empleo como ayudante de especialistas en pegar azulejo en las construcciones de casas y edificios. Es decir, tomar un segundo empleo implicaba para Luis tener una doble jornada, por la mañana en la compañía de materiales de construcción y en la tarde y noche en el restaurant, donde aprendió a cocinar comida rápida. Posteriormente, 
después de 18 meses de desempeñarse en ambos empleos, el restaurant cerró sus puertas y se quedó laborando en un solo empleo, pero de manera paralela tomaba trabajos temporales por una jornada o una semana, y mantenía el empleo en la compañía. Los últimos 8 meses trabajó como empleado en una compañía que realizaba reparaciones de casas y edificios, donde previamente ya había trabajado, antes de ir a Atlantic City.

Durante la estancia de seis años en Estados Unidos, Luis tuvo una incursión por dos meses y medio en Atlantic City, donde trabajó por un mes en un restaurant chino (como ayudante de cocina) y un mes y medio como empleado general en una compañía de instalación de cocinas integrales. Este viaje lo realizó cuando tenía 5 años residiendo en Nueva York, entonces decidió probar suerte en otro lugar.

Finalmente, después de dos meses y medio fuera de Nueva York y de constatar que percibía un sueldo menor en Atlantic City (aproximadamente $40 \%$ menos que en NYC), decidió volver a Nueva York. Allí se empleó por 8 meses con un patrón puertorriqueño con quien ya había trabajado y estuvo como empleado hasta que decidió volver a Nezahualcóyotl. La decisión de regresar la tomó a finales de 2002 porque recibió un video donde sus padres le mostraron la casa casi concluida y entonces eso lo motivó a volver, pues había alcanzado la meta propuesta. Paralelamente, un evento familiar (la muerte de una sobrina en NYC) le hizo reflexionar sobre el momento de su regreso. Así tomó la decisión de volver, trayendo consigo ahorros y el plan de establecer un negocio. Regresó a Nezahualcóyotl y estuvo un mes y medio sin trabajar (por decisión propia) hasta que finalmente regresó a la misma compañía de embutidos, en el puesto de obrero general, el mismo en el que se desempeñaba antes de viajar a Nueva York.

Luis fue entrevistado en el mes de febrero de 2011, entonces tenía 3 años de haber retornado a Nezahualcóyotl, y estaba haciendo planes para reemigrar a Estados Unidos un mes más tarde, con el objetivo de trabajar por dos años en la ciudad de Nueva York para luego volver a Nezahualcóyotl. Contaba con los recursos económicos para financiar el viaje y planeaba realizarlo llevando a un amigo primerizo (primer viaje a Estados Unidos) del municipio de Los Reyes La Paz, un municipio vecino en la zona conurbada del Distrito Federal.

Caso 2: ROBERTO (entrevistado en mayo de 2012 en Nezahualcóyotl) 48 años, casado, retornado de Estados Unidos en enero de 2010, después de vivir en aquél país por 20 años. Actualmente es trabajador por cuenta propia, tiene un pequeño taller de carpintería y herrería en la colonia La Perla, en Nezahualcóyotl, localizado a dos casas de la casa de su madre, donde él y su familia residen. Roberto es un inmigrante de Michoacán en Nezahualcóyotl, a 
donde llegó con sus padres a la edad de 4 años, procedente de un pequeño pueblo del estado de Michoacán. Ocupa el lugar 5 de 13 hermanos. Viajó en 1990 a California a la edad de 28 años, específicamente a Long Beach donde vivía una hermana que estaba casada y trabajaba como baby sitter en aquella ciudad. Roberto vivía con su hijo de cuatro años y su esposa, los tres miembros de la familia emigraron y realizaron juntos el viaje.

En Nezahualcóyotl, Roberto trabajaba como empleado en una fábrica, donde hacía trabajo de herrería, eventualmente también carpintería, ambos oficios los había aprendido con sus hermanos y padre. Una de las motivaciones para emigrar no fue la falta de empleo, asegura que "nunca conoció en Neza la carestía de trabajo", sino un evento que lo desmoralizó: le robaron la herramienta de carpintería que había llevado a la fábrica donde trabajaba para realizar una labor especial. Decide entonces emigrar apoyado por su hermana quien financió el viaje a Long Beach, Cal. en octubre de 1990, pero la decisión fue tomada por Roberto y su familia en junio del mismo año, es decir consideró cuatro meses para no abandonar de inmediato su empleo.

En el periodo de veinte años que vivió en Estados Unidos no realizó ningún viaje a Nezahualcóyotl (es decir, a México), de tal forma que Roberto ha realizado en su vida un viaje al interior del país cuando se mudó del estado de Michoacán a Nezahualcóyotl, y un solo viaje de México a Estados Unidos cuando emigró a aquél país.

Roberto y su familia cruzaron la frontera en Tijuana y llegaron por tierra a Long Beach, California, vivieron con la hermana y su familia en un espacio pequeño donde además habitaban al menos seis personas más, entre primos y sobrinos. Asegura que por primera vez en su vida "sentí que no podía conseguir un empleo". Llegó a principios del mes de octubre de 1990 y consiguió su primer empleo a finales de enero de 1991, después de obtener documentos tales como el social security number y la green card falsos. Su primer empleo fue como ayudante en una tintorería, en la cual trabajaba su cuñado; en este sitio laboró como empleado durante un año y medio, planchando ropa. En ese periodo pasó de la posición de ayudante a empleado general (planchador), cuando su cuñado llegó a ser el encargado de la tintorería. Luego de un año y medio en Long Beach se mudó al estado de Washington, muy cerca de Seattle, lugar al que se movieron los patrones de su hermana y junto con ellos, la hermana decidió emigrar con toda la familia, incluidos Roberto y los suyos. Allá se empleó nuevamente en una tintorería, como planchador, donde laboró por siete años y medio aproximadamente. En el mismo sitio su esposa trabajó hasta 1999, también por 7 años, como empleada, encargada de doblar las prendas y etiquetarlas, pero nunca en la atención al público. 
Roberto decidió tomar otro empleo, después de 7 años y medio en la tintorería, en donde renunció por problemas de salud, debido a la exposición constante a las altas temperaturas. Se empleó como obrero en una fábrica de ventanas y aluminio, en donde por sus conocimientos y habilidades adquiridas como herrero en Nezahualcóyotl, rápidamente aprendió el oficio de "aluminero" (especialista en trabajar el aluminio). Trabajó por 10 años en esa fábrica (1999-2009), haciendo uso siempre de sus documentos apócrifos. En 2009 se enfrentó a dos dilemas vitales: a) la expiración de la tarjeta que contenía el número de seguridad social y adicionalmente de su green card que le permitían ser contratado en la fábrica, no obstante que los patrones sabían que eran falsos - suponía Roberto. b) Su esposa había sido diagnosticada con una enfermedad crónica algunos años antes y para entonces se había agravado su condición. Adicionalmente, en 2005 habían experimentado una crisis familiar que le llevó a Roberto a acercarse, y más tarde afiliarse como miembro, con los Testigos de Jehová, también por intermediación de su hermana, quien se había convertido a esa religión años atrás. Después de 4 años de converso, Roberto enfrentó el dilema "moral y ético" - así lo llama - de si renovaba sus documentos de la misma forma que los había obtenido, o bien declaraba en la fábrica que los documentos eran falsos y se sometía a la decisión del patrón, de ser contratado o no sin documentos. Tomó la segunda opción y fue despedido en los primeros meses de 2009.

Durante 2009 consiguió solamente empleos eventuales por la falta de documentos, por un día, una semana o si acaso 15 días continuos. En 2010 empeoró la condición de su esposa y como ya no tenía un empleo con seguridad social, decidió pedir ayuda a la familia de la esposa que radicaba en Iztapalapa, D.F. Entonces él y su hijo decidieron también volver a Nezahualcóyotl en diciembre de 2010 junto con la esposa.

Regresó a México con una camioneta, seis mil dólares, y herramienta para realizar trabajo tanto de carpintería como de herrería; aunque la mayor parte de esa herramienta y de las técnicas aprendidas no las ha podido implementar, por ejemplo técnicas relacionadas con la fabricación de portones/zaguanes debido a que en Nezahualcóyotl se "acostumbra" colocar portones en las entradas de las viviendas, "prácticamente blindados". Cuenta con herramienta que tiene un valor comercial importante, tales como tornos y maquinaria con la que podría fabricar en serie sillas con tubulares. No obstante, Roberto decidió poner un pequeño taller de carpintería y herrería, que se localiza en la cochera de la casa de un hermano, a dos casas del lugar que actualmente habita. Adicionalmente, durante su estadía en Estados Unidos compró una casa en la periferia de Seattle, en un terreno de dos acres y medio, la cual aparentemente vendió antes de viajar de regreso a México, pero no ha recibido aún el pago. 
Roberto fue entrevistado en mayo de 2012, a solo un año y seis meses de haber retornado a Nezahualcóyotl, en donde de inmediato empezó a trabajar como carpintero y herrero. Asimismo se ha insertado en un Salón del Reino de los Testigos de Jehová localizado en la colonia donde reside. Ha enfrentado algunas dificultades porque lo ven "diferente" comparativamente con la imagen que proyectaba en el momento de su partida, hace 20 años, ahora con una nueva religión y otra forma de relacionarse con los vecinos, "más respetuosa y distante" por no compartir sus festividades religiosas y cívicas. Asimismo, su madre y demás familiares - así lo afirmó Roberto - reconocen que no se integra igual en las celebraciones con la familia porque no consume alcohol, aunque hizo el esfuerzo de pasar a saludar personalmente a las familias que viven en la calle donde residía antes de viajar, la misma donde actualmente reside; algunos lo recordaban, otros no lo conocían. Reconoce que observa muchos cambios en Nezahualcóyotl, ocurridos en 20 años, los lugares se han transformado, las calles ahora lucen pavimentadas, ha crecido la colonia (en términos espaciales, y poblacionales por supuesto) y se han agregado varias nuevas, entre otros cambios que registra. Asegura que no volverá a Estados Unidos, no obstante que su sueño era regresar siendo "un inmigrante legal" y no sin documentos, pero no consiguió esa meta.

\section{Un balance de los casos prototípicos}

Los casos prototípicos fueron construidos entre un conjunto de trayectorias de migrantes de retorno reciente en Nezahualcóyotl (ambos inmigrantes retornaron en los últimos cinco años), representan casos prototípicos de "reinserción con reemigración" y "reinserción con establecimiento". Estos prototipos permiten observar las condiciones en las cuáles ciertos eventos familiares y personales definen el momento del retorno, pero no sin estar mediado por las condiciones del mercado laboral en los lugares de destino. Asimismo, sus experiencias de reinserción social y laboral son producto de la intersección entre lógicas locales/familiares y globales/transnacionales.

En el caso del prototipo "inserción con establecimiento" se identifica nítidamente cómo la experiencia personal se intersecta con las condiciones históricas como inmigrante indocumentado en Estados Unidos; es decir, la enfermedad de la esposa, sumada a la falta de documentos migratorios y la decisión personal de no obtenerlos nuevamente falsos, tiene como consecuencia para Roberto, el enfrentar una situación difícil para la consecución de un nuevo empleo, una vez que es despedido de la fábrica; y luego confinarse, en la última etapa de su vida en Estados Unidos, a tomar empleos de bajo salario, corto plazo, ninguna certidumbre laboral, y de baja calificación, no 
obstante contar con una amplia experiencia como obrero en fábricas y maquiladoras en Estados Unidos, habiendo desarrollado habilidades como herrero, carpintero y aluminero durante al menos una década de trabajo en aquél país, y una década adicional en Nezahualcóyotl, previo a su viaje internacional.

Pero no solo la decisión del retorno fue motivada por esta intersección de eventos familiares y la dinámica del contexto y las condiciones actuales de la inmigración indocumentada en Estados Unidos, particularmente en el contexto de la crisis de los mercados laborales que han contribuido en la implementación de una política aún más restrictiva en materia de derechos y oportunidades para los inmigrantes en el último quinquenio; sino también la experiencia de retorno a Nezahualcóyotl y el proceso de reinserción tanto social como laboral han sido moldeados en esta lógica. Su afiliación en 2005 al grupo religioso de los Testigos de Jehová y su involucramiento religioso durante los años posteriores influyeron fuertemente en una serie de decisiones relevantes de su vida como inmigrante, como trabajador y como persona. El retorno es visto también - desde la mirada de Roberto -, como una resultante de la elección de una "forma ética y moral de vida". Su reinserción social en Nezahualcóyotl ha estado ligada no a la posibilidad de reactivar las viejas redes familiares y vecinales, sino a la oportunidad de construir y activar una nueva red de relaciones a partir de introducirse en un grupo de los Testigos de Jehová, cuyo establecimiento se localiza en la colonia donde él reside actualmente. Su inserción religiosa le ha permitido reinsertarse socialmente, en un lugar donde después de 20 años de ausencia, se percibe a sí mismo diferente, y observa cómo ha cambiado también el lugar y las personas que lo habitan. En este caso su inserción está mediada sin duda por la dinámica de las religiones globales que han alcanzado las localidades y han diversificado las opciones religiosas, que también innegablemente implican otras formas de vida y de relación con la otredad. Es decir, el efecto de su conversión religiosa en Estados Unidos ha permeado su inserción social (y en algunos momentos también su inserción laboral) en Nezahualcóyotl; lo mismo que su inserción laboral en Estados Unidos estuvo también influida por su experiencia previa de trabajo en la carpintería y la herrería en Nezahualcóyotl.

La lógica entre reinserción y establecimiento entonces está mediada por la dinámica entre mercados de trabajo y modalidades de inserción social creadas a partir de la intersección de la experiencia vital e histórica de los inmigrantes en esta localidad y su relación con los habitantes nativos en ese espacio, sin perder de vista que tales están atravesadas por la dinámica global y la posición que en esa escala ocupa la localidad, y luego el contexto de 
retorno. Por esa razón, la experiencia de retorno urbano y rural es diferenciada, debido a la estructura de oportunidades en la que un inmigrante o retornado se insertan y la posibilidad de transformar tales estructuras o de diseñar mecanismos que posibiliten su inserción, reinserción, o establecimiento, así como también la re-emigración a un destino internacional, o bien uno nacional.

Por otro lado, el caso prototípico construido para dar cuenta de "inserción con reemigración" hace posible delinear otras vías de inserción social a partir del hecho de que el retornado ocupa la posición de hijo en el hogar, y vuelve a reinsertarse en la dinámica familiar del hogar paterno, y luego a retomar una posición en el empleo que ya había ocupado previamente a su viaje internacional. Cómo opera en este caso el capital acumulado y qué papel tiene en su reinserción tanto social como laboral su experiencia de vida como inmigrante internacional en la localidad de Nezahualcóyotl, para que finalmente Luis decida reemigrar a Estados Unidos.

Estas son algunas de las interrogantes que se apuesta pueden ser respondidas a través de esta investigación aun en ciernes. Así, se vislumbra a través de nuestros hallazgos, la condición de incertidumbre en la cual está ocurriendo el retorno migratorio internacional en este contexto urbano metropolitano en México.

\section{Referencias}

ALARCÓN, Rafael. El retorno de los migrantes mexicanos. La Jornada, 28.10.2008, p. 4. CASSARINO, Jean-Pierre. Theorising Return Migration: The Conceptual Approach to Return Migrants Revisited. International Journal on Multicultural Societies, v. 6, n. 2, 2004, p. 253-279.

DURAND, Jorge. Ensayo teórico sobre la migración de retorno. El principio del rendimiento decreciente. Cuadernos geográficos, v. 35, n. 2, 2004, p. 103-116.

DURAND, Jorge; MASSEY, Douglas. Clandestinos. Migración México-Estados Unidos en los albores del siglo XXI. México: Miguel Ángel Porrúa Librero Editor, 2003.

ESPINOZA, Víctor M. El dilema del retorno, género y pertenencia en un contexto transnacional. México: El Colegio de Jalisco y El Colegio de Michoacán, 1998.

GLICK SCHILLER, Nina. Transnational social fields and imperialism: Bringing a theory of power to Transnational Studies. Anthropological Theory, v. 5, n. 4, 2005, p. 439-461.

. Beyond the Nation-State and its Units of Analysis: Toward a New Research Agenda for Migration Studies. Essentials of Migration Theory. Working Paper, n. 33, 2007, Center on Migration, Citizenship and Development, p.1-42. 
GLICK SCHILLER, Nina; CAGLAR, Ayse (eds.). Locating Migration, Rescaling Cities and Migrants. Ithaca, New York: Cornell University Press, 2011.

GLICK SCHILLER, Nina; FAIST, Thomas (eds.). Migration, Development and Transnationalization. A Critical Stance. New York: Berhahn Books, 2010.

INEGI - INSTITUTO NACIONAL DE ESTADÍSTICA, GEOGRAFÍA E INFORMÁTICA. Censo General de Población y Vivienda, 2000. México: INEGI, 2000.

. Conteo de población 2005. México: INEGI, 2005.

. Censo General de Población y Vivienda, 2010. México: INEGI, 2010.

LEVITT, Peggy; SØRENSEN, Ninna N. The transnational turn in migration studies. Global Migration Perspectives, n. 6, 2004, p. 2-13.

LEVITT, Peggy; GLICK-SCHILLER, Nina. Conceptualizing Simultaneity: a Transnational Social Field Perspective on Society. International Migration Review, v. 38, n. 3, 2004, p. 1002-1040.

LINDSTROM, David P. Economic Opportunity in Mexico and Return Migration from the United States. Demography, v. 33, n. 3, 1996, p. 357-374.

MASSEY, Douglas; GOLDRING, Luin; DURAND, Jorge. Continuities in Transnational Migration: An Analysis of Nineteen Mexican Communities. American Journal of Sociology, v. 99, n. 6, 1994, p. 1492-1533.

PAPAIL, Jean. De asalariado a empresario: La reinserción laboral de los migrantes internacionales en la región centro-occidente de México. Migraciones Internacionales, v. 1, n. 33, julio-diciembre 2002, p. 79-102.

Remesas e inversiones de los migrantes de retorno en el centro-occidente de México. In DELGADO WISE, Raúl; KNERR, Beatriz (coords.). Contribuciones al análisis de la migración internacional y el desarrollo regional en México. México: Miguel Ángel Porrúa Librero Editor, 2005, p. 319-332.

PEDREÑO-CÁNOVAS, Andrés. Familias inmigrantes: el trabajo de los padres y las estrategias de trabajo de los hijos en las áreas mediterráneas de agricultura intensiva. In LARA FLORES, Sara (coord.). Migraciones de trabajo y movilidad territorial. México: Miguel Angel Porrúa Librero Editor, 2010, p. 333-365.

RIVERA SÁNCHEZ, Liliana. El eslabón urbano en el trayecto interno del circuito migratorio Mixteca-Nueva York-Mixteca: Los migrantes de Nezahualcóyotl, Estado de México. In LEVINE, Elaine (ed.). La migración y los latinos en Estados Unidos. Visiones y Conexiones. México: Universidad Nacional Autónoma de México, 2008, p. 53-73.

. ¿Quiénes son los retornados? Apuntes sobre el migrante retornado en el México contemporáneo. In FELDMAN-BIANCO, Bela et alii. La construcción social del sujeto migrante en América Latina. Prácticas, representaciones y categorías, Quito: Facultad Latinoamericana de Ciencias Sociales y Consejo Latinoamericano de Ciencias Sociales, 2011, p. 309-338.

. Las trayectorias en los estudios de migración. Una herramienta para el análisis longitudinal cualitativo. In ARIZA, Marina; VELASCO, Laura (coords.). 
Métodos cualitativos y su aplicación empírica. Por los caminos de la investigación sobre migración internacional. México: Universidad Nacional Autónoma de México y El Colegio de la Frontera Norte, 2012, p. 455-494.

RIVERA-SÁNCHEZ, Liliana; LOZANO ASCENCIO, Fernando. Los contextos de salida urbanos y rurales y la organización social de la migración. Migración y Desarrollo, n. 6, primer semestre, 2006, p. 45-78.

SINGER, Paul. Economía política de la urbanización. México: Siglo XXI Editores, 1975.

STARK, Olson. The Migration of Labour. Cambridge: Basil Blackwell, 1991.

TAYLOR, J. Edward. Development strategy, Employment and Migration: Insight from Models. Paris: OECD Development Center, 1996.

TODARO, Michael P. A model of Labour Migration and urban employment in less development countries. The American Economic Review, v. 59, n. 1, 1969, p. 138-148.

ZAHNISER, Steven S. One Border, Two Transitions: Mexican Migration to the United States as a Two-Way Process. American Behavioral Scientist, n. 42, 1999, p. 1350-1380.

\section{Abstract}

\section{Return Migration and Re-insertion Experiences in the Metropolitan Area of Mexico City}

This article analyzes the contemporary conditions of returning of Mexican immigrants from the U.S. to Mexico, particularly in urban contexts. Firstly, it analyzes some demographical data of return population from the National Census of Population, 2000 and 2010. Secondly, it presents some biographical narratives about return experiences of people going back during the last decade, with the aim of offering empirical evidence about which are the conditions of social and labor re-insertion, how they are processing the re-emigration (international/domestic) or the settlement, and how such actors create their relocation strategies in urban contexts in Mexico.

Keywords: return migration, social and labor re-insertions, U.S., Mexico.

Recebido para publicación en 18/08/2013.

Aceptado para publicación en 14/11/2013.

Received for publication in August, 18 ${ }^{\text {th }}, 2013$. Accepted for publication in November, 14 ${ }^{\text {th }}, 2013$.

ISSN impresso: 1980-8585

ISSN eletrônico: 2237-9843 